Q Nueva Etinexaxio Revista Digital de OFilosofia ISSN 1850-3578 2013 - Vol. 8 - Número VIII - Resistencia, Chaco, Argentina. Pp. 125 - 135

\title{
El suplicio de Tántalo y la circularidad de las búsquedas. Una mirada a la búsqueda en los entornos digitales
}

\author{
Víctor Manuel Reyes Espino \\ Reynaldo Thompson \\ Universidad de Guanajuato (México)
}

Recibido: $25 / 07 / 2013$

Aceptado: $19 / 08 / 2013$

\section{Resumen}

El hombre, entonces es definido como un ser de búsquedas, mismas que a la vez se definen por motivaciones y circunstancias personales o contextuales las que pueden incluir en gran medida construir, preservar, consolidar o, por el contrario confrontar estructuras de poder. Sin embargo, ¿podríamos encontrarnos con realidades animadas que no sean movidas por el deseo de encontrar algo? ¿las búsquedas en sí mismas se habrán constituido en un "objeto" que permita consolidar una nueva forma de control colectivo? El objeto de éste trabajo es el de indagar sobre estas interrogantes, enfatizando sobre los matices que la búsqueda ha tomado a partir de la consolidación de entornos digitales especializados influyendo de manera decidida en la emergencia de un nuevo fenómeno de búsqueda virtual donde el proceso se consolida como fin y el deseo de encontrar se concentra en un ejercicio placentero de un movimiento circular infinito.

Palabras clave: Virtualidad, entornos digitales, buscadores, búsqueda, sociedad

\begin{abstract}
The man, then, is defined like a searching been, same that by the way are defined by motivations and personal or contextual circumstances the ones that can be mostly included as build, preserve, consolidate, or the opposite, confront the power's structures. But ¿can we found animated realities that haven't been motivated for wish find something? ¿does the search in its own have been conformed themselves in an object that can let consolidate a new way of collective control? The objective of this work is investigate about this questions, making
\end{abstract}


QNueva Ettinexaxia Revista Digital de OFilosofia ISSN 1850-3578 2013 - Vol. 8 - Número VIII - Resistencia, Chaco, Argentina. Pp. 125 - 135

emphasis in the ways that the search had toke since the consolidation of specialized digital environments influencing in a strong way at the emergency of the new virtual searching phenomenon were the process is consolidated like an objective and the wish of find is concentrated in a pleasant exercise of an endless circular movement.

Keywords: virtuality, digital environments, search engines, search, society. 


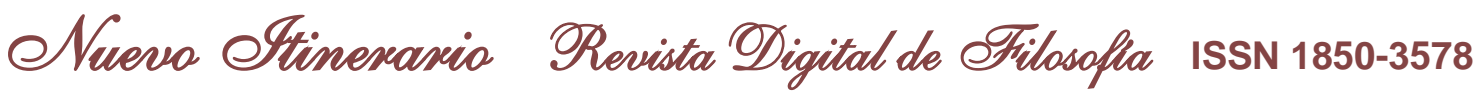
2013 - Vol. 8 - Número VIII - Resistencia, Chaco, Argentina. Pp. 125 - 135

Lo inanimado y lo animado son realidades complejas, situación que nos llevaría a formular una construcción por exclusión. Lo inanimado excluye lo animado (o viceversa) y dicha exclusión se distingue, entre otras cosas, por la capacidad o incapacidad de moverse. Lo animado tiene movimiento per se, lo cual está profundamente vinculado a otra realidad: el fin, el cual a su vez se encuentra impulsado por algo que se necesita, o en estados más, elaborados, algo que se desea; en suma, algo que se busca.

El hombre, entonces es definido como un ser de búsquedas, mismas que a la vez se definen por motivaciones y circunstancias personales o contextuales las que pueden incluir en gran medida construir, preservar, consolidar o, por el contrario confrontar estructuras de poder. La gama se extiende por múltiples matices que van desde las búsquedas expresadas en grandes mitos, hasta las manifiestas en caminos que se consolidan por la vía de la razón y método. Las que se han movido por el camino de la estética y la expresión artística hasta las que fundado su razón en la constitución del poder por el poder mismo.

Sin embargo ¿podríamos encontrarnos con realidades animadas que no sean movidas por el deseo de encontrar algo?¿existiría la posibilidad de un movimiento que no tenga como fin el encontrar sino el sólo placer de ser o estar?. O en otro orden, ¿̇las búsquedas en sí mismas se habrán constituido en un "objeto" que permita consolidar una nueva forma de control colectivo? Si es así, ¿de qué manera las búsquedas que impulsan la creación artística son influenciadas por estas plataformas especializadas que orientan las inquietudes y las decisiones que mueven al artista? El objeto de éste trabajo es el de indagar sobre estas interrogantes, enfatizando sobre los matices que la búsqueda ha tomado a partir de la consolidación de entornos digitales especializados influyendo de manera decidida en la emergencia de un nuevo fenómeno de búsqueda virtual donde el proceso se consolida como fin y el deseo de encontrar se concentra en un ejercicio placentero de un movimiento circular infinito.

\section{Espacialidad y temporalidad, del deambulante al buscador.}

El buscar implica hacer lo necesario para encontrar, y lo necesario implica transitar entre espacios y tiempos definidos permitiendo ubicar con certeza lo encontrado. Así la dimensión espacio temporal constituye el entramado profundo sobre el que se desarrolla el ejercicio y definición de las búsquedas mismas que influyen de manera decidida en las intencionalidades, los porqué ligados al deseo del encuentro. Estas intencionalidades se definen ante el objeto de búsqueda por un anhelo de aprehensión, posesión, deconstrucción o simplemente contemplación 


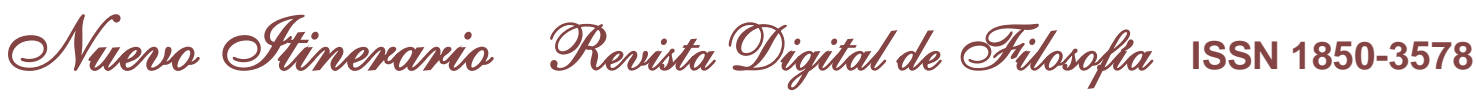
2013 - Vol. 8 - Número VIII - Resistencia, Chaco, Argentina. Pp. 125 - 135

Ramírez Kuri citando a Walter Benjamin en sus reflexiones sobre Baudelaire, nos describe "un paseante, urbano , consumidor, neurasténico y algo dandi" ${ }^{1}$, un fláneur o deambulante que plácidamente transita por espacios urbanos sin la intención de detenerse, pero sí de apropiación de los espacios urbanos que transita

El tránsito de espacios que implicaban un deambulaje no exigía necesariamente un deseo de búsqueda, era un tránsito placentero cuyas rutas eran predispuestas por espacios físicos inamovibles y que eran seguidos durante el caminar del deambulador.

La búsqueda, a diferencia del deambulaje placentero implica una ruta definida por el deseo de encontrar, las motivaciones subjetivas de completar lo inconcluso, donde la ausencia se hace presente, y mueve a completar con presencias, la búsqueda es regida por un movimiento para completar lo incompleto, "todo lo que ocurre como significativo ocurre en el horizonte de otras posibilidades. Este es el caso de otras posibilidades que (...) son negadas y lo que ocurre se introduce como necesario" ${ }^{2}$ Cuando la búsqueda implica incidir en la temporalidad, deteniendo el devenir informativo emerge la perspectiva crítica.

\section{Del mito de Tántalo a las búsquedas como fetiches.}

En la mitología griega existe la historia de Tántalo, rey de Frigia y amigo de los dioses. Éste se distinguía por tratar de gratificar siempre a los inmortales, aunque en el fondo sentía un profundo desdén hacia los mismos. Así fue que en una ocasión organizó un gran banquete, para lo cual asesinó y descuartizó a uno de sus hijos ofreciéndolo como platillo principal. Ninguno de los dioses, sospechando del desequilibrio de Tántalo, probó bocado excepto Ceres, que sin darse cuenta probó el alimento dándose cuenta de que era carne humana. Esta suerte de sacrilegio con el que desafiaba y ofendía a los dioses, le mereció un castigo ejemplar, se le condenó a vivir inmerso en un lago a la sombra de árboles frutales, de ésta manera viviría en una eterna sed y hambre, incapaces de ser saciadas; lo más que aspiraría el condenado es a humedecer sus labios, que al contacto con el agua, se resecarían más acrecentando cada vez su deseo de beber. Así, Tántalo sueña con comer y beber de lo que está a su alcance; sin embargo, nunca los podrá alcanzar por más que se esfuerce.

La metáfora se constituye como la representación perfecta del buscador en los laberintos de los artilugios y creaciones digitales, el Tántalo que transita por la red en búsqueda de saciar su sed desde lo que en apariencia está a su alcance; sin embargo al tocar sus labios con la

\footnotetext{
${ }^{1}$ RAMIREZ Kuri, Patricia (et al), Pensar y habitar la ciudad, afectividad, memoria y significado en el espacio urbano contemporáneo España, Cuadernos A Temas de Innovación Social, Universidad Autónoma Metropolitana 1ạ. Ed. , 2006. P.147

2LUHMANN, N “Tiempo y mundo e historia sistémica” en Inguruak Junio , 1999, pp 13-54
} 


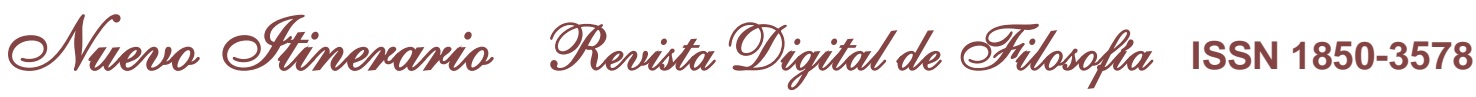
2013 - Vol. 8 - Número VIII - Resistencia, Chaco, Argentina. Pp. 125 - 135

infinitud de la información, sólo logra acrecentar el deseo, el anhelo. La ausencia demandante de las presencias.

Sin duda que los origenes del buscador digital se pueden trazar en el flaneur analizados por Walter Benjamin en el inacabado Libro de los Pasajes en el que el flaneur parisino estaba seducido por un mundo de mercancias, mientras que para el Tántalo, el buscador se encuentra inmerso en la información, en la fuente que puede saciar su sed, pero le es imposible apropiarse de ella, detenerse y dejar de fluir, la crítica le es imposible, y mientras no modifique su condición, como el condenado, nunca podrá alcanzar lo que busca por más que se esfuerce, al contrario el deseo y la necesidad de completarse se ven acentuadas reafirmando su condición de buscador. Entonces las búsquedas se tornan circulares, sin principio ni fin, en una navegación indefinida sin posibilidades de detenerse, de reflexionar.

En este círculo, o tal vez mejor dicho, espiral de ausencia, la búsqueda puede llegar a tomar la condición de mercancía, el producto perfecto que vende pero no exige retribución sino el deseo de ahondar en la insatisfacción y la frustración de lo que se desea. El síndrome tantálico se convierte así en el fetiche perfecto, para que desde artilugios tecnológicos se enfatice en las ausencias, acrecentado el deseo de lo infinito, ese "infinito que sólo se realiza y se despliega a través de la superación, la substitución de un ser finito por otro. El movimiento mediante el cual lo finito lleva en sí, dada su naturaleza, el principio de su propia destrucción”³.

Herbert Marcuse ya desde hace tiempo intuía que la insatisfacción creada se constituía en la estrategia de precondicionamiento con miras a desarrollar formas y medios de control colectivo que más tarde facilitarían el camino a los artilugios masivos como medios de masificación y anulación de los individuos. Dicha insatisfacción se desarrollaría a partir de un estrechamiento en el lindero entre lo dado y lo posible.

El precondicionamiento no empieza con la producción masiva de la radio y la televisión y con la centralización de su control. La gente entra en esta etapa ya como receptáculos precondicionados desde mucho tiempo atrás; la diferencia decisiva reside en la disminución del contraste (o conflicto) entre lo dado y lo posible, entre las necesidades satisfechas y las necesidades por satisfacer ${ }^{4}$.

Sin ello la sociedad actual sería inconcebible reafirmando el deseo insaciable de consumo instaurándose la búsqueda como mercancía cultural en la que nos sometemos al

\footnotetext{
${ }^{3}$ GARAUDY Roger, El pensamiento de Hegel , España, Ed. Seix Barral, 1974, p. 18

${ }^{4}$ MARCUSE, Herbert. El hombre unidimensional. España, Ed. Ariel Filosofía: 2010. p. 47
} 
Pverea Etinexaxio Revista Digital de OFilosofla ISSN 1850-3578 2013 - Vol. 8 - Número VIII - Resistencia, Chaco, Argentina. Pp. 125 - 135

perfeccionamiento del despilfarro, en "donde las formas predominantes del control social son tecnológicas en un nuevo sentido "Así el buscador se convierte en un consumidor, proyectando ante sí sus ausencias y constituyendo a la búsqueda como un fenómeno fetichista, es decir,

La cosificación de las relaciones sociales, la personificación de los objetos creados por el trabajo humano, la inversión entre sujetos y objetos, la cristalización del trabajo social global en una materialidad objetual que, como espectro social, aparenta ser autosuficiente y poseer vida por sí misma - por ejemplo el equivalente general que devenga interés-, la coexistencia de la racionalidad de la parte con la irracionalidad del conjunto y la fragmentación de la totalidad social en segmentos inconexos. ${ }^{6}$

Esa personificación de las búsquedas, las colocan en la posibilidad de mercancía, y los buscadores en posición de objetos consumidores, así la búsqueda en entornos digitales, convertida en fetiche es caracterizado

Por congelar y cristalizar cualquier proceso de desarrollo, definiendo discursiva o ideológicamente alguna instancia de lo social como si fuera fija cuando en la vida real fluye y se transforma. Las relaciones sociales se "evaporan" súbitamente y su lugar es ocupado por las cosas, las únicas mediadoras de los vínculos intersubjetivos a nivel social. La aparente "objetividad absoluta" de la estructura social termina predominando por sobre las subjetividades sujetadas al orden fetichista. Las reglas que rigen la vida de esa objetividad que escapa a todo control humano cobran autonomía absoluta y toman el timón del barco social. Se vuelven independientes de la conciencia y la voluntad colectivas. ${ }^{7}$

En suma, el suplicio de Tántalo radica en la incapacidad de saciar su sed y su hambre a pesar de estar en contacto con la fuente de su satisfacción, suplicio que se infringe a quién busca, desde los artilugios digitales impuestos desde las estructuras de poder para administrar la fuente de satisfacción, saciar su anhelo circular, eternamente inacabado.

\footnotetext{
${ }^{5}$ Idem. p. 48

6 KOHAN , Néstor, "Fetichismo y poder en la teoría crítica contemporánea" [en línea] 2011 [citado 2011-11-20]. D: http://www.nodo50.org/agora/files/files/kohan-feti.pdf .

${ }^{7}$ Idem. p.2
} 


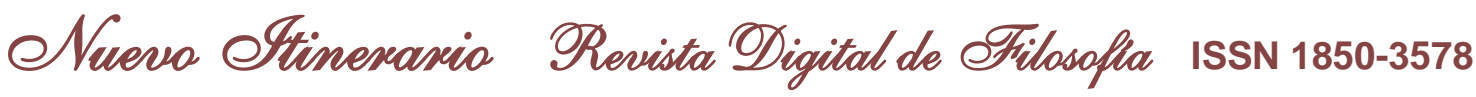
2013 - Vol. 8 - Número VIII - Resistencia, Chaco, Argentina. Pp. 125 - 135

\title{
Del placer de buscar a la estética de la simulación.
}

Sin embargo, ¿podríamos advertir alguna posibilidad estética en el acto de búsqueda a través de los artilugios digitales? ¿advertir la posibilidad de placer estético en el sujeto que busca? Las primeras aproximaciones tendríamos que elaborarlas desde una reflexión de los nuevos procesos desde donde se construye la percepción del mundo en el que se es.

Un punto de partida es la dependencia tecnológica que el hombre orgánico contemporáneo ha desarrollado; en este sentido

\begin{abstract}
Se afirma que las prótesis mediadas por computador desarrolladas desde mediados del siglo XX como extensiones tecnológicas de nuestro cuerpo biológico, han afectado profundamente nuestra capacidad de imaginación y alterando nuestra relación espacio- temporal con las cosas. Esto se refiere en la forma como vemos al mundo y a nosotros mismos y, consecuentemente, esto transforma el modo como proyectamos y construimos nuestra vida ${ }^{8}$
\end{abstract}

Esta afectación, sobre todo en su percepción espacio - temporal define su posición ante la búsqueda; Deleuze y Guattari, citados por Landow, proponen que el navegador dentro de los entornos digitales, es un

Ser para el espacio, en vez de ser en el espacio. Estamos en el agua, inscribiendo y siendo inscritos por la corriente en nuestro navegar. Nos escribimos en oscilación entre el tranquilo espacio del ser para el tiempo (lo que nos ocurre cuando avanzamos así como lo que le ocurre al espacio en el que nos movemos) y el espacio estriado del "en tiempo" (lo que ocurre fuera del espacio y de nosotros) ${ }^{9}$

El ser para el espacio en el tiempo, ¿podría generar una sensación de libertad donde el lector puede escoger su propia experiencia, sin estar ligado o limitado dentro de ninguna organización o jerarquía ${ }^{10}$ ?. Al revisar las estructuras de poder que determinan las direcciones

\footnotetext{
8 MICHELIN Simone “Dominio público”, en HERNÁNDEZ García lleana (comp.), “Estética, ciencia y tecnología. Creaciones electrónicas y numéricas", Colombia, Ed. Pontificia Universidad Javeriana, 2005, (Colección Biblioteca del profesional. Línea de Estética contemporánea) p. 202

9 LANDOW George P., "Hipertexto 3.0. Teoría crítica y nuevos medios en la era de la globalización”, Ed. Paidos Comunicación, EUA, 2006, p.91

${ }^{10}$ Idem., p.90
} 
Pverea Etinexaxio Revista Digital de OFilosofla ISSN 1850-3578 2013 - Vol. 8 - Número VIII - Resistencia, Chaco, Argentina. Pp. 125 - 135

de este hiperespacio nos ubicarían en una primera aproximación a un no, ya que las construcciones y sensaciones estarían enmarcadas en una experiencia de la simulación orientada y predeterminada para favorecer intereses de los sujetos sociales en el poder.

En un experimento realizado por Landow y Kahn, con la finalidad de detectar las dificultades de movilidad en hipertextos, registraron que "un usuario al "hacer clic accidentalmente" en un determinado enlace encontró que había tomado un "delicioso desvío", puesto que le proporcionó una solución para uno de los problemas"11. Curiosa la expresión "delicioso desvió", la cual nos hace inferir la emergencia de un arquetipo cuyo eje rector permite calificar como placentero el "desviar", el apartar a una persona o cosa de su destino o del camino o dirección que sigue, haciéndole seguir otro distinto; o el de disuadir de un propósito, intención o dictamen que se había propuesto ${ }^{12}$. El placer de encontrar caminos de búsqueda distintos a los propuestos inicialmente.

En este contexto, conviene rescatar la propuesta de lo que Giannetti denomina como la estética de la simulación, en ésta el buscador (observador) ha sido transformado

\begin{abstract}
En interactor interno, que participa en un modelo de mundo construido artificialmente, juegan con la impresión de que la persona puede introducirse más allá de la interfaz humano - máquina (...). En este tipo de mundo simulado, los observadores internos se mueven en dos realidades; la realidad de su conciencia que participa en un juego de simulación, y la realidad de su percepción que le indica que su presencia y conducta tienen influencia activa en el mundo artificial, de manera que las distorsiones peculiares a su observación se reflejan y se producen en el entorno en el que se encuentra inmerso ${ }^{13}$
\end{abstract}

Bajo esta perspectiva, ahondamos en la complejidad sobre la que se construye la experiencia estética del navegador donde las dos realidades de conciencia y percepción se encuentran inmersas en la lógica de lo que pretendo ser; la lógica de la simulación. Giannetti suma a este concepto el de endoestética, en la cual "el interactor desempeña una función dentro de la

\footnotetext{
${ }^{11}$ Idem., p.271

12 Larousse Editorial, S.L. 2006.

${ }^{13}$ GIANNETTI, Claudia "Estéticas de la simulación como endoestética” , en Op. Cit. HERNÁNDEZ García, p. 92
} 


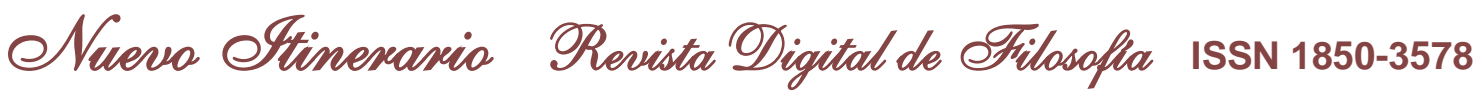
2013 - Vol. 8 - Número VIII - Resistencia, Chaco, Argentina. Pp. 125 - 135

obra, comparte una experiencia espaciotemporal en el interior del sistema. La obra se presenta como una simulación del mundo peculiar, como un endosistema."14

De esta manera regresamos al mito del Tántalo, donde el infortunado rey se mueve en la lógica de una necesidad consciente y que no puede ser satisfecha a pesar de estar inmerso en el entorno que podría colmar su deseo, y la inmersión misma se instaura como el recordatorio de la imposibilidad de una insatisfacción colmada. De ésta misma manera el buscador, atrapado en las redes del endosistema creado por el exosistema del poder,

\section{De endosistema a ecosistema, implicaciones desde la circularidad..}

Partimos del supuesto que el buscador se encuentra inmerso en un camino marcado por la circularidad, infinitud motora en que busca colmar los anhelos que un sistema propone como infinitos con fines de preservación de la estructura que le otorga sentido. Sin embargo, ¿podría dicha situación tener algún impacto directo en el sistema, exosistema, que contiene y sostiene las redes sobre las que se construye el endosistema? Probablemente sí, Guattari ${ }^{15}$ propone que en estos contextos de fragmentación surgen nuevas problemáticas ecologistas donde los modos de vida deben cambiar radicalmente desde la forma de las relaciones humanas, con la naturaleza, sino incluso al interior de la sociedad Para Guattari el cambio sugerido no puede venir únicamente desde la perspectiva del medioambientalismo sino desde un abandono de los hábitos consumistas promovidos por el sistema en un mundo que se desquebraja al tratar de satisfacer una opulencia infinita.

El sujeto no es evidente, no basta pensar (...) puesto que muchas otras formas de existir se instauran fuera de la conciencia, mientras que cuando el pensamiento se empeña obstinadamente en aprehenderse a sí mismo, (...) sin captar ninguno de los territorios reales de la existencia, los cuales, por su parte, deriva los unos en relación con los otros ${ }^{16}$

Gamboa - Bernal proponen la construcción de una ecología humana que permita reflexionar no sólo las relaciones entre sistemas de seres vivos, sino las relaciones de orden simbólico y cultural

\footnotetext{
${ }^{14}$ Idem. p. 93

${ }^{15}$ GUATTARI Felix. Las Tres Ecologías,, España ,ed. Pre-textos, España, 1996. P. 51

${ }^{16}$ Idem. p.22
} 
Q Nueva Etinexaxio Revista Digital de OFilosofla ISSN 1850-3578 2013 - Vol. 8 - Número VIII - Resistencia, Chaco, Argentina. Pp. 125 - 135

En la ecología general se estudian las relaciones entre los ecosistemas vivientes, mientras que la ecología humana tiene también la perspectiva de las relaciones interpersonales y culturales. Si con el descuido de la primera ecología se producen crisis ambientales, con la negligencia en la ecología humana se llega a trances mucho más graves: crisis humanas y desastres culturales, como los que estamos viviendo hoy. ${ }^{17}$

El ensimismamiento ahonda una búsqueda infructuosa, sin sentido, donde la lógica de la insatisfacción, de los deseos eternos y etéreos, impacta en la configuración de las relaciones con el otro, en sus construcciones simbólicas que le permiten desarrollar un imaginario impregnado de individualismo normando para su subsistencia la anulación del otro, trastocando el alma del entramado social abonando a una crisis humana donde los territorios existenciales se comienzan a dislocar y construirse sobre simulaciones teñidas de insatisfacción.

Transitar a una ecología "centrada en el cuidado y la administración del mundo, empezando por el hombre mismo, que haga posible una renovación cultural profunda"18, con nuevas posibilidades que permita la reconstrucción de imaginarios redireccionando el sentido de sus deseos y orientados sus búsquedas.

De lo anterior derivamos que romper el ciclo tantálico en que se encuentra inmerso el hombre moderno exige una nueva concepción ecológica, entendida coma la reconfiguración de relaciones sociales y de construcción subjetiva orientada no a sistemas cerrados sobre un sí mismo, sino abierto a territorios existenciales y procesuales "a partir de praxis que permitan hacerlo habitable por un proyecto humano"19.

\footnotetext{
${ }^{17}$ GAMBOA-Bernal, Gilberto A “Ecología humana ecología ambiental binomio clave" Persona Y Bioética, 15(1), 5-9.

18 Idem. p. 2

${ }^{19}$ Ob. Cit. GUATTARI Felix. Las Tres Ecologías. p 52
} 
ENueva Otinenaxio Revista Digital de Prilosofla ISSN 1850-3578 2013 - Vol. 8 - Número VIII - Resistencia, Chaco, Argentina. Pp. 125 - 135 